\title{
ARTICLE
}

Cite this: DOI: 10.1039/x0xx00000x

Received 00th January 2012, Accepted 00th January 2012

DOI: $10.1039 / \times 0 x \times 00000 x$

www.rsc.org/

\section{Magneto-plasmonic nanoparticles as theranostic platforms for magnetic resonance imaging, drug delivery and NIR hyperthermia applications}

\author{
Inmaculada Urries ${ }^{\dagger}$, Cristina Muñoz ${ }^{\dagger}$, Leyre Gomez ${ }^{\dagger}$, Clara Marquina ${ }^{\mathrm{a}, \mathrm{b}}$, Victor \\ Sebastian $^{\dagger *^{*}}$, Manuel Arruebo ${ }^{\dagger *}$ and Jesus Santamaria ${ }^{\dagger * *}$
}

PEGylated magneto-plasmonic nanoparticles with a hollow or semi-hollow interior have been successfully synthesized and their physico-chemical characteristics investigated. The hollow interior space can be used to store drugs or other molecules of interest whereas magnetic characterization shows their potential as contrast agents in magnetic resonance imaging (MRI) applications. In addition, their plasmonic characteristics in the near infrared (NIR) region make them efficient in photothermal applications producing high temperature gradients after short irradiation times. We show that by controlling the etching conditions the inner silica shell can be selectively dissolved to achieve a hollow or semi-hollow interior without compromising the magnetic or plasmonic characteristics of the resulting nanoparticles. Magnetic measurements and transmission electron microscopy observations have been used to demonstrate the precise control during the etching process and to select an optimal concentration of etching reagent and contact time to preserve the inner superparamagnetic iron oxide-based nanoparticles and the plasmonic properties of the constructs. Drug loading capabilities were also evaluated for both semi-hollow and as-synthesized nanoparticles using Rhodamine B isothiocyanate as a model compound. The nanoparticles produced could be potentially used as "theranostic" nanoparticles with both imaging capabilities and a dual therapeutic function (drug delivery and hyperthermia).

\section{Introduction}

The knowledge gained in recent years regarding gold nanostructures allows a precise control of their shape, size and composition and is promoting a wider use of these nanomaterials in biomedical applications., ${ }^{1}$ Gold nanostructures present good biocompatibility, low toxicity and easy bioconjugation. ${ }^{1}$ The surface plasmon absorption (SPA) of $\mathrm{Au}$ nanostructures provides remarkable optical properties that enable their use as diagnostic and therapeutic nanoplatforms. This SPA is highly dependent on the surface, shape, size, ligand, inter-particle distance and dielectric properties of the nanoparticle and of the surrounding medium. In particular, if a gold nanoshell is grown on a dielectric material (e.g. silica) the SPA can be tuned in the near infrared region (NIR) of the electromagnetic spectrum by tailoring the core and shell dimensions. ${ }^{3}$ Regarding biomedical purposes, the NIR region between 700-1100 $\mathrm{nm}$ is preferable because water and biological chromophores present very low absorbance or scattering at those wavelengths, allowing potential applications in localized photothermal therapy 4,5 or modulated drug delivery. ${ }^{6,7}$ In thermal therapy, NIR absorbed radiation is converted efficiently into heat. This phenomenon is caused by electron-phonon and phonon-phonon processes and allows the use of Au nanostructures in photothermal therapy. ${ }^{8,9}$

Superparamagnetic iron oxide nanoparticles (SPIONs) can exhibit high magnetization under external magnetic fields and no remanence nor coercivity. These characteristics make SPIONs suitable for magnetic fluid hyperthermia, for directing carried biomolecules to tumours using magnetic field gradients, as well as contrasts agents in magnetic resonance imaging (MRI). ${ }^{10}$ MRI is a powerful imaging technique that does not use ionizing radiation. A radio frequency (RF) pulse at the frequency capable of transferring energy to water protons in body tissues is applied, and upon the RF pulse removal, the magnetic moments of the protons relax to equilibrium in a determined time called relaxation time, which is tissuedependent. $T_{1}$ is the time constant of the exponential recovery process of the longitudinal magnetization after a RF pulse. $T_{2}$ is the time constant of the exponential decay of the transversal magnetization. Consequently, the differences in $T_{1}$ or $T_{2}$ values in a tissue generate the corresponding contrast in MRI. In some tissues where differences in $\mathrm{T}_{1}$ and $\mathrm{T}_{2}$ are small, contrast agents 
are used to modify those times and achieve a better contrast image. The efficiency of a contrast agent can be expressed by the relaxivity $(\mathrm{r} 1, \mathrm{r} 2)$ defined as the slope of the plot $1 / \mathrm{T}_{1}$ or $1 / T_{2}$ as a function of the magnetic material concentration, according to Equation 1.

Equation $1 \quad R_{1,2}=R_{1,2}^{\circ}+r_{1,2} \times C$

Where $R_{1,2}\left(s^{-1}\right)$ is the respective $1 / T_{1}$ or $1 / T_{2}$ proton relaxation rate in the presence of the contrast agent, $\mathrm{R}_{1,2}$ are the relaxation rates in the absence of contrast agent and $\mathrm{C}$ is the contrast agent concentration (mM). The constant of proportionality $r_{1,2}\left(\mathrm{~s}^{-1} \cdot \mathrm{mM}^{-1}\right)$ is the above mentioned relaxivity and measures how much of the proton relaxation rate is increased per unit of concentration of contrast agent. ${ }^{11}$ Regarding the efficiency of contrast agents for imaging applications, the higher the relaxivity, the better the imaging sensitivity achieved. ${ }^{11}$

SPIONs have a high transverse relaxivity, which enables their application as $T_{2}$ contrast agents as they shorten the $T_{2}$ relaxation time of protons. These particles have been widely studied and several formulations have been approved by the FDA ${ }^{12-15}$ to image liver cancer, although some have been discontinued due to safety or market issues ${ }^{16}$. Therefore, SPIONs with clinically relevant characteristics remain to be further developed. For biomedical applications water solubility, colloidal stability and nonspecific protein adsorption on SPION surfaces are a must. SPIONs should be surface functionalized as naked SPIONs are rapidly recognized and cleared by the rethiculoendothelial system. Different coatings are being studied including $\mathrm{SiO}_{2}$, biomolecules or polymers, $\mathrm{Au}$, dextran, polyethylene glycol (PEG), peptides and protein shells, and the coating influence in $T_{2}$ and $r_{2}$ has also been thoroughly studied. ${ }^{17-19}$

Finally, $\mathrm{SiO}_{2}$ is widely applied as coating material on SPIONs because the silica shell protects the core from the environment avoiding agglomeration, providing colloidal stability and allowing simple and direct biofunctionalization using a well establish silane-based chemistry. Different authors have reported the strong influence of the silica shell thickness in the nanoparticle efficiency as MRI contrast agent. ${ }^{20-22}$

Multifunctional nanostructures that combine magnetic and optical properties have been reported in the literature (see figures A-D in scheme 1). Several groups have attempted the synthesis of multifunctional core-shell nanostructures with a gold shell, having plasmon absorption in the NIR region, and a superparamagnetic iron oxide-silica core, with SPIONs between the silica core and gold shell ${ }^{23,24}$ (see scheme 1-a) or SPIONs inside silica cores ${ }^{25,26}$ (see scheme 1-b). Also, silver has been grown on $\mathrm{Au}$ nanoshells, yielding a sandwich-like nanostructure where the plasmon absorption has been shifted across a wide wavelength range by tuning the Ag shell coverage and thickness (see scheme 1-c) ${ }^{27}$. Other materials like organic polymers have been used as dielectric interfaces between magnetic iron oxide cores and $\mathrm{Au}$ shells ${ }^{28}$ (see scheme 1-d). We have followed a different approach to develop, to the best of our knowledge, novel nanostructures using the SPION-gold structures of scheme 1-b, as starting point to finally develop novel hollow structures (scheme 1-e,f). Excellent reviews about bifunctional magneto-optical nanostructures and their potential applications can be found in the literature 29,30 . Targeted drug delivery is the process of releasing a bioactive agent at a specific site in a controlled manner. Many systems based on nanoparticles as carriers are being investigated for this purpose including polymeric, solidlipid, ceramic, magnetic, metal-based NPs, dendrimers and polymeric micelles or vesicles ${ }^{31-33}$.
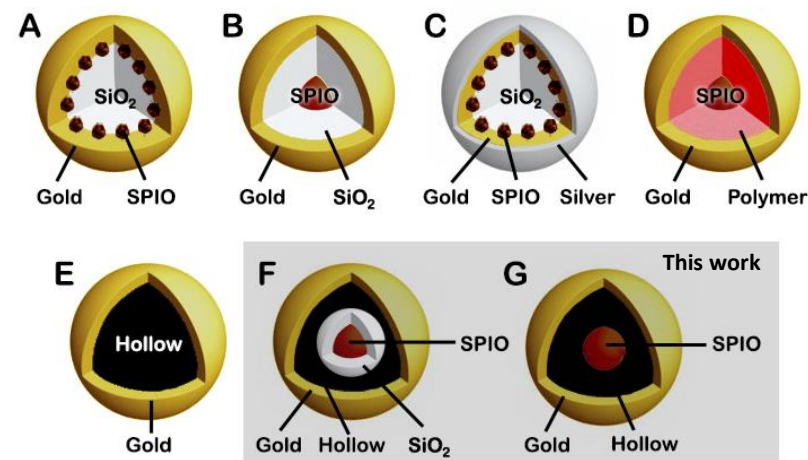

Scheme 1.- A-E: Summary of gold-shell magnetic nanoparticles reported in the literature. Magnetoplasmonic nanoparticles engineered in this work: F) Partially hollow magnetic nanoshells. G) Hollow magnetic nanoshells.

An ideal nanocarrier should contain the maximum drug load, target specifically the desired site without releasing the drug prematurely and follow the desired release kinetics depending on the envisaged pathology. ${ }^{34}$

Hollow gold nanoshells present a thin Au wall with a hollow interior $^{35}$ (see scheme 1-e). These structures combine both the optical properties of gold nanoshells (large absorption in the NIR region) and the possibility of physically entraping drugs in their hollow interior. Drugs may be loaded by encapsulation inside the hollow structure or by surface attachment where drugs are conjugated to the inner or outer gold surfaces using labile bonds, ${ }^{36}$ although in this case the amount of conjugated drug is limited. The payload may be increased by using a porous core of high surface area. ${ }^{7}$

SPION-gold based nanoparticles have also been explored for drug release applications. Examples of doxorubicin (DOX) loading by adsorption on solid NPs have been reported by several authors. $^{32}$ In the case of porous hollow gold nanospheres it has been also demonstrated that it is possible to load a higher quantity of DOX due to their larger surface area than on solid nanoparticles for the same gold content. ${ }^{36}$ The resulting nanostructures could be applied in image-guided therapy where a drug could be delivered on demand by activation with optical hyperthermia, using the heat to trigger release by accelerating desorption of the loaded drug or by breaking a thermally sensitive bond.

Taking the above into account, we propose in this work a new type of multi-functional nanoparticles which fill the gap existing in the reported gold nanostructures (see Scheme 1). We have attempted the synthesis of two types of nanoparticles 
with optical, magnetic and drug loading properties by engineering continuous but porous $\mathrm{Au}$ nanoshells on hollow/partially hollow nanostructures containing magnetic cores (see scheme 1 f-g).

\section{Results and discussion}

\section{Hybrid (double shell) nanoparticle formation}

The synthesis of the magneto-plasmonic nanoparticles is a multi-step procedure (see scheme 2), where an excellent control on the silica-shell growth is needed, to allow its subsequent homogenous etching resulting in monodisperse hollow magnetic nanoshells. When this is not achieved, a mixture of hollow and partially hollow magnetic nanoshells results. Also, a homogeneous particle-size distribution is required with similar $\mathrm{SiO}_{2}$ shell thicknesses on the SPIONs, since otherwise during etching the thinnest shells would dissolve sooner leaving the magnetic cores exposed to chemical attack. The concentration of SPIONs was optimized to tune the $\mathrm{SiO}_{2}$ shell thickness. The higher the concentration of SPIONs, the lower the $\mathrm{SiO}_{2}$ shell thickness, since the TEOS amount was kept constant. Figure 1-a shows the appearance of the SPIONs used as cores in the initial step of the synthesis. Figure 1-b and c show the monodisperse $\mathrm{SiO}_{2}$-SPIONs obtained, with particle distributions that could be narrowly tuned in the range between $93 \pm 10 \mathrm{~nm}$ and $167 \pm 19 \mathrm{~nm}$ (see Table 1). It can be seen that the SPIONs-SiO ${ }_{2}$-consist of a magnetic core (dark contrast) encased by a dense silica layer. Several SPIONs were entrapped inside the silica. TEM characterization revealed the absence of free SPIONs and also the presence of at least one SPION inside each silica particle, showing the selective growth of $\mathrm{SiO}_{2}$ on the SPION surface.

Figure 1d shows the appearance of the $\mathrm{SPION}-\mathrm{SiO}_{2}$ particles after seeding with Au nanoparticles, from which a continuous



Scheme 2.- Schematic illustration of the formation of hollow $(\mathrm{H}) /$ partially-hollow (PH) etched SPION-nanoshells. A silica shell was grown on SPIONs, which was then surface functionalized to replace silanol (Si-OH) by amino groups using silanization with APTES. Tiny Au nanoparticles were anchored to the surface of functionalized silica and a gold continuous shell (nanoshell) was grown using a gold hydroxide solution and formaldehyde. Nanoshells were PEGylated to promote biocompatibility and avoid aggregation in further steps. $\mathrm{NaOH}$ or $\mathrm{BHF}$ were selected to control the $\mathrm{SiO}_{2}$ etching and to tune the volume of the cavity inside the nanoshell, leading to hollow or partially hollow SPION-nanoshells.
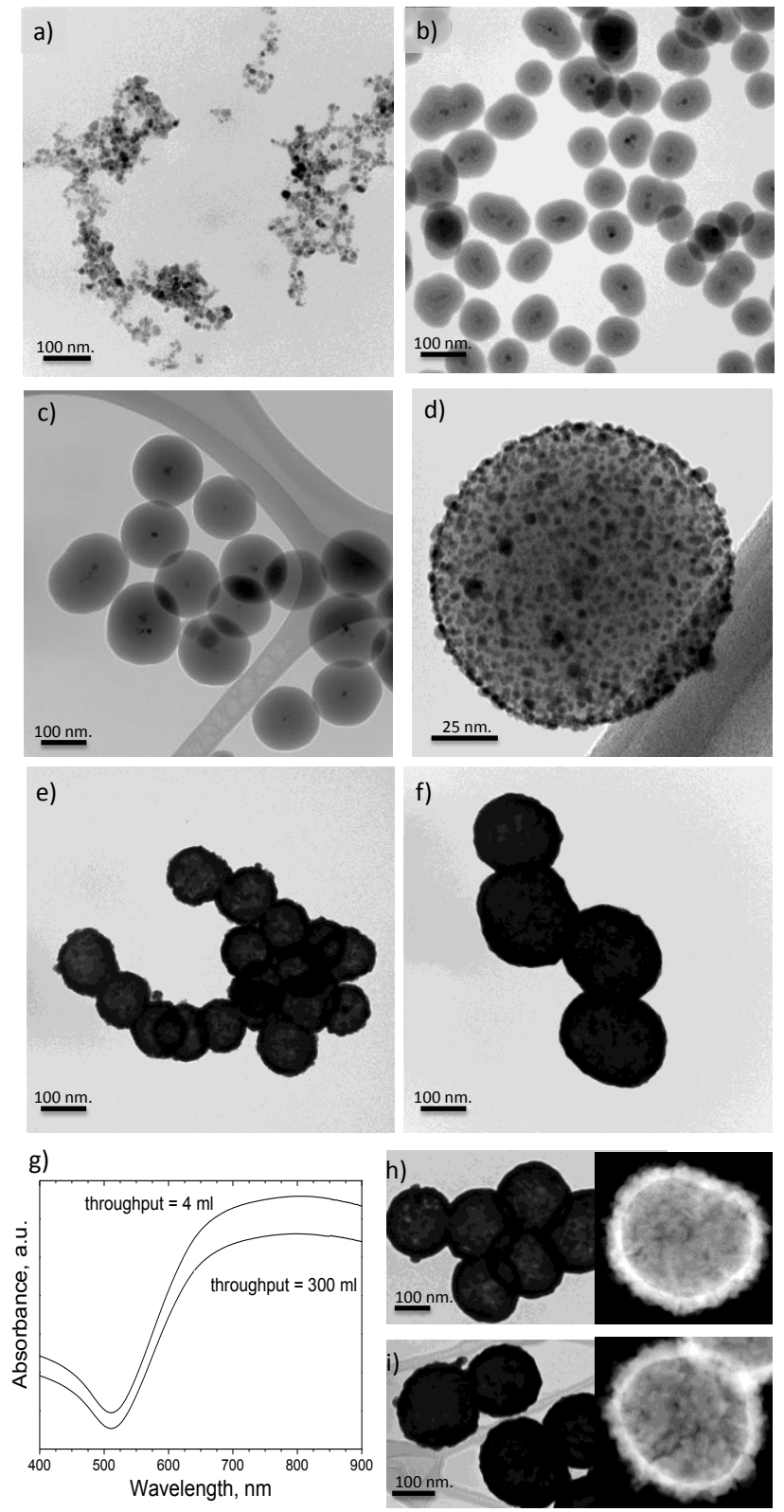

Figure 1.- Transmission electron micrographs. a) SPIO nanoparticles with a particle diameter of $11,8 \pm 5 \mathrm{~nm}$. SPION-SiO 2 nanoparticles (NPs) with a different SiO2 shell thickness: b) particle size $93 \pm 10 \mathrm{~nm}$, c) particle size $167 \pm 19 \mathrm{~nm}$. d) Detail image of a SPION-SiO ${ }_{2}-\mathrm{Au}$ NPs, obtained after Au seeding. Nanoshells, SPION-SiO2-Au NPs, obtained after Au seeding and Au shell growth on SPION$\mathrm{SiO}_{2}$ : e) obtained from particles in fig 1-b, particle size $125 \pm 11 \mathrm{~nm}, \mathrm{f}$ ) obtained from particles in fig 1-c, particle size $202 \pm 25 \mathrm{~nm}$. g) UV-VIS spectra of the asmade magnetic nanoshells (S) obtained under a throughput of $4 \mathrm{~mL}$ and $300 \mathrm{~mL}$ per batch. Transmission electron micrographs (left: TEM and right: STEM-HAADF) of the nanoshells obtained at different throughputs: h) $4 \mathrm{~mL}$, i) $300 \mathrm{~mL}$.

gold shell will be grown (Figures 1e-1f). As reported by Oldenburg et al. ${ }^{3}$, the Au seeds attach to the silica surface, increase in size and then coalesce forming islands until finally an apparently continuous metallic nanoshell is formed. The images (Figures 1-h, 1-i) show that the gold coating is continuous but porous, with topographical roughness at the nanometer scale. A proper gold nanoshell growth is required because if the nanoshell is not continuous or if it is too thin, its 
mechanical stability would be compromised after $\mathrm{SiO}_{2}$ etching. In addition, a thick solid nanoshell without nanometric pinholes or inter-crystal gaps would not allow the diffusion of etchants through the $\mathrm{Au}$ nanoshell to create a hollow interior. The amount of Au deposited on the silica particles was tuned by changing the ratio of Au-seeded SPION-silica cores to the total amount of gold in the hydroxide solution added during nanoshell growth.

In the last synthesis step (see Scheme 2), the magnetic nanoshells were coated with PEG because of its demonstrated biocompatibility. We found that the PEGylation step was highly necessary to preserve the nanoshells colloidal stability over time after the etching step. Whereas non-PEGylated nanoshells exhibited a strong tendency towards aggregation after removal of the etchants, PEGylated nanoshells showed no visible signs of aggregation and were easily dispersed without the need of ultrasonication.

The corresponding Au shell thicknesses and average diameter of the silica cores together with the corresponding core/shell dimension ratios for all synthesized $\mathrm{SPION}-\mathrm{SiO}_{2}-\mathrm{Au}$ nanostructures $(\mathrm{S} 1, \mathrm{~S} 2, \mathrm{~S} 3)$ are presented in Table 1. According to the data, the thinner the nanoshell, the higher the blue-shift of the surface plasmon resonance (SPR) peak. It must be stated that prior to shell growth, each sample exhibits a plasmon extinction peak at $520 \mathrm{~nm}$ that corresponds to the $\mathrm{Au}$ seeds (results not shown). The SPR ranges from $750 \mathrm{~nm}$ in S1 to 854 $\mathrm{nm}$ in S3. These tendencies agree with the predictions based on a Drude model. ${ }^{37}$ Theoretical calculations have shown that the plasmon resonance of gold metal nanoshells can vary over hundreds of nanometers in the VIS-Near IR range, as the position of the plasmon resonance is directed by both the shell thickness and the size of the dielectric core. ${ }^{38}$

Scaling-up the production of nanoshells with the desired dimensions is a major issue to assure the viability and reproducibility of biomedical experiments. ${ }^{39}$ Fortunately in this case the process could be simply scaled up by a factor of 75 (from 4 to $300 \mathrm{~mL}$ ) by increasing the reaction volume while keeping the proportions of the reactants and avoiding the use of magnetic stirrers. Figure 1-g shows the UV-VIS spectra from both types of nanoshell productions. From this figure it is inferred that the optical properties of the nanoshells resulting

Table1.-Morphological properties of the $\mathrm{Au}$ nanostructures obtained: assynthesized nanoshell (S), partially-hollow nanoshell (PH) and hollow nanoshell $(\mathrm{H}) .1,2,3$ stands for particles obtained with a different $\mathrm{SiO}_{2}$ shell thickness

\begin{tabular}{|c|c|c|c|c|}
\hline NPs type & $\begin{array}{c}\mathrm{SiO}_{2} \text { dia., } \\
\mathrm{nm}\end{array}$ & $\begin{array}{c}\text { NS-dia., } \\
\mathrm{nm}\end{array}$ & $\begin{array}{c}\text { Au shell } \\
\text { thk., } \mathrm{nm}\end{array}$ & $\begin{array}{c}\mathrm{SiO}_{2} \text { Core/Au Shell } \\
\text { ratio }\end{array}$ \\
\hline $\mathrm{S} 1$ & $93 \pm 10$ & $125 \pm 11$ & 16 & 2.9 \\
\hline $\mathrm{PH} 1^{\text {b) }}$ & $68 \pm 20$ & $125 \pm 11$ & 16 & 2,2 \\
\hline $\mathrm{S} 2$ & $137 \pm 13$ & $178 \pm 18$ & 20.5 & 3.3 \\
\hline $\mathrm{PH} 2^{\text {b) }}$ & $124 \pm 14$ & $178 \pm 18$ & 20.5 & 3,0 \\
\hline $\mathrm{S} 3$ & $167 \pm 19$ & $202 \pm 25$ & 17.5 & 4.8 \\
\hline $\mathrm{PH}^{\text {b) }}$ & $96 \pm 33$ & $202 \pm 25$ & 17.5 & 2,7 \\
\hline $\mathrm{H}^{\text {a) }}{ }^{\text {b }}$ & - & $202 \pm 25$ & 17.5 & - \\
\hline
\end{tabular}

a) NaOH Etching (15 minutes). b) BHF etching (2 hours). dia. = diameter; thk. = thickness from both syntheses were maintained even though the synthesis production was increased by nearly two orders of magnitude. The morphological study of the nanoshells obtained according to both production scales shows that the mean particle size and surface topography were preserved (see Figure 1h-i). In particular STEM images (on the right hand side of Figure $\mathbf{1} \mathbf{h}$-i) also shows that the $\mathrm{Au}$ nanoshell thickness $(17.5 \mathrm{~nm})$ is not affected by the scaled-up production.

\section{Etching of the inner silica shell (H and PH nanoshells)}

The BHF solution is a buffered hydrofluoric acid etching solution. It has good etch-rate consistency and uniformity and is commonly used for oxide removal as part of microfabrication processes. The presence of $\mathrm{NH}_{4} \mathrm{~F}$ controls the $\mathrm{pH}$ and enables the steady replenishment of fluoride ions to maintain a stable etch rate. BHF promotes the anisotropic wet-etching, which implies that there is a directional etching with different etching rates in different directions. The Au inter-crystal defects created during the nanoshell growth allow the BHF diffusion and the formation of cavities in the $\mathrm{SiO}_{2}$ that eventually turn the solid nanoshell into a hollow nanostructure.

Following the etching of $\mathrm{SiO}_{2}$ under the $\mathrm{Au}$ shell by transmission microscopy is challenging because the Au shell has a contrast higher than that of the inner $\mathrm{SiO}_{2}$ core. In this work we propose both the $\mathrm{T}_{2}$ relaxation times and the relaxivity of water suspensions of these synthesized nanoparticles as indicators to follow the $\mathrm{SiO}_{2}$ etching since the thickness of the silica and the SPION content influence those parameters. ${ }^{21,22,}$ ${ }^{40}$ Figure 2 depicts the influence of the BHF concentration added to the suspension on the proton tranverse relaxation rate, $1 / T_{2}$, and TEM images obtained when etching with different BHF concentrations during $2 \mathrm{~h}$. Each data point in the curve corresponds to a test performed on the nanoshell suspension

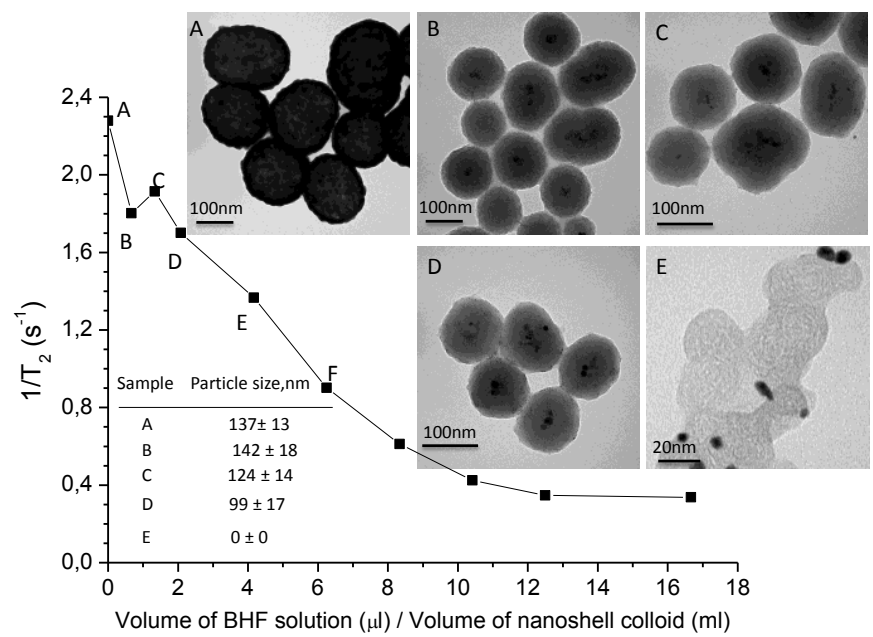

Figure 2.- Transverse relaxation rate $\left(1 / T_{2}\right)$ as a function of the degree of silica etching using a BHF solution. Insets show: Transmission electron micrographs and particle size of Au nanoshells at different silica etching. The TEM images, named from $A$ to $E$, are correlated with the transverse relaxation data in the graph. Observation: Gold shell was selectively removed by an aqua regia treatment in order to visualize the inner $\mathrm{SPION}-\mathrm{SIO}_{2}$ core and gain knowledge about the etching. 
obtained after adding a determined concentration of BHF, in which $\mathrm{T}_{2}$ has been measured and a TEM image obtained. The TEM images were taken after the treatment of the resulting nanoshells with aqua regia, which leads to the selective etching of $\mathrm{Au}$ to visualize the $\mathrm{SiO}_{2}$ etching process the nanoshells. $\mathrm{T}_{2}$ measurements are directly comparable in all tests since they were performed with the same initial SPION concentration. Any variation of $T_{2}$ implies a change in $r_{2}$ (see Equation. 1). A decrease in $1 / T_{2}$ can be ascribed to reduction in the SPIO concentration caused by an (unwanted) etching of The increase in the BHF content has a direct effect on the etching rate, promoting the formation of soluble $\mathrm{Si}$-based species (see Figure 2). Simultaneously, the transverse relaxation rate decreases when increasing the BHF concentration.. The particle sizes (and therefore the thicknesses of the $\mathrm{SiO} 2$ shell) obtained from the TEM images of the samples etched using different amounts of BHF correlate well with transverse relaxation rates measured after each etching step. Consequently, BHF etches the $\mathrm{SiO}_{2}$ shell under the $\mathrm{Au}$ shell, but it also etches the SPION cores, as the decrease in the transverse relaxation rate suggests (all the samples were washed before each T2 measurement and therefore, the eventual contribution of iron ions in the solution to $\mathrm{T}_{2}$ was negligible).

Our experiments point to a critical thickness of the $\mathrm{SiO}_{2}$ shell below which it is not able to protect the SPION core against a liquid etchant such as BHF solution and therefore the magnetic core completely dissolves. To confirm this assumption, an uncontrolled anisotropic etching was carried out, consequence of which porous $\mathrm{SiO}_{2}$ nanoparticles around $28 \mathrm{~nm}$ were formed, containing no SPIONs (see TEM image $E$ in Figure 2). As expected, the measured transverse relaxation rate of this nanoparticle suspension was close to that of pure water. These results suggest that $\mathrm{BHF}$ promotes the formation of corrosion pits or pores in the silica phase, providing effective paths for the diffiusion of fluoride ions that are able to reach and etch the inner SPION nanoparticles according to Equation 2 (see Experimental). However, under controlled conditions, the BHF approach enables only a partial etching of the silica shell, preserving the magnetic cores.

Figure 4-a shows a nanoshell etched to obtain a partially hollow structure. The STEM images (Figure 4-a,b) show that the Au shell is stable after the BHF treatment. A gold shell thickness of approximately $17 \mathrm{~nm}$ can be observed with a high brightness contrast. The elemental profile along the nanoshell volume obtained by EDS-HAADF analysis shows the location of a $20 \mathrm{~nm}$ SPION core inside the nanoshell (see Figure 4-b-d). The Si elemental profile also shows a $60 \mathrm{~nm} \mathrm{SiO}_{2}$ shell protecting the SPION core. The location of the SPION is slightly shifted from the nanoshell center. This location is statistically more feasible in partially hollow nanostructures than in hollow ones.

An alternative wet-etching approach using $\mathrm{NaOH}$ was proposed to enable the formation of a completely hollow nanoshell while preserving the interior SPION, a structure which could be also named as a magnetic $\mathrm{Au}$ nanorattle. Figure 3 depicts the modification of the

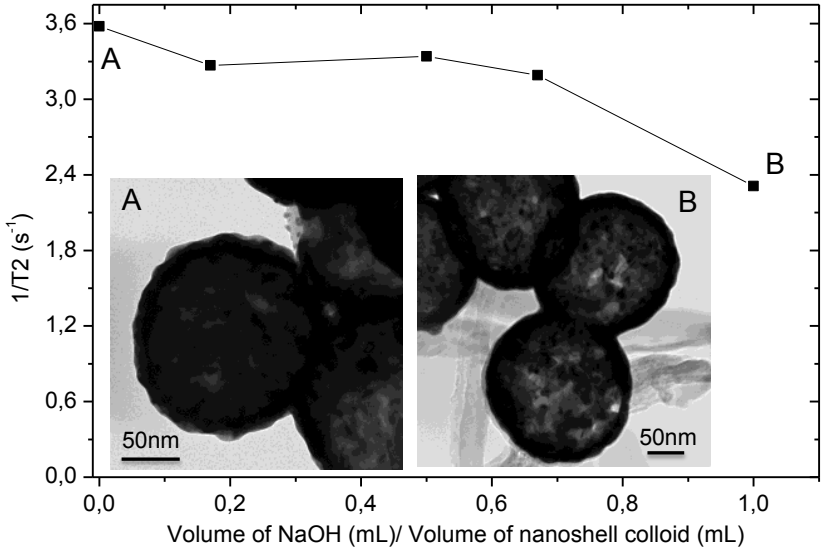

Figure 3.- Transverse relaxation rate $\left(1 / T_{2}\right)$ as a function of the degree of silica etching using a $\mathrm{NaOH}$ solution $3 \mathrm{M}$. Transmission electron micrographs of $\mathrm{Au}$ nanoshells at different silica etching stage: A) non-etched $\mathrm{SiO}_{2}$ core. B) completely etched $\mathrm{SiO}_{2}$ core. The TEM image shows different contrast at different shell locations. This is attributed to the presence of pores or pinholes in the $\mathrm{Au}$ shell. These pinholes where observed after the $\mathrm{SiO}_{2}$ shell was removed by etching.

transverse relaxation rate as the $\mathrm{NaOH}$ content was increased in the etching process. Although Equation 4 (see Experimental) shows that $\mathrm{NaOH}$ can also react with $\mathrm{Fe}$, it seems that the etching of the SPIONs is less pronounced since the $1 / \mathrm{T}_{2}$ variation is considerably less significant than that obtained by $\mathrm{BHF}$ etching. In fact, the transverse relaxation rate of water was not achieved (which would mean the complete disappearance of the SPIONs), at least for the highest $\mathrm{NaOH}$ concentration used in our experiments. The TEM image B in Figure 3 shows a hollow magnetic nanoshell where defects in the Au shell play the role of pores and can only be observed once the $\mathrm{SiO}_{2}$ material supporting the Au shell was completely etched. The size of these pores on the $\mathrm{Au}$ nanoshell is smaller than the SPION dimensions, so the SPIONs cannot leak out of the $\mathrm{Au}$ nanoshell cage. Figure 4-e shows a STEM micrograph of a hollow magnetic nanoshell. The $\mathrm{Au}$ shell is not damaged because of the $\mathrm{NaOH}$ treatment, but its mechanical stability is not as high as the dense (i.e. with the un-etched $\mathrm{SPION}-\mathrm{SiO}_{2}$ core) nanoshell. The location of the SPIO nanoparticles inside the hollow nanoshell requires a careful study by EDS-HAADF analysis since the magnetic core can be located anywhere in this nanorattle structure. According to the EDS-HAADF spectra and the elemental mapping of the area selected in figure 4-e,f, the SPION was located close to the Au nanoshell. There was no trace of $\mathrm{SiO}_{2}$ protecting the magnetic cores and therefore, it is clear that the nanostructure is hollow. This observation was also confirmed by the $\mathrm{Au}$ elemental profile along the particle diameter: the Au signal intensity is higher in the external area corresponding with the particle surface, where upper and lower wall are observed through the empty void of the particle (see figure 4-g). The Fe elemental profile also confirms the presence of Fe close to the Au nanoshell. No intensity coming from $\mathrm{SiO}_{2}$ was measured in the profile. Consequently, the $\mathrm{SiO}_{2}$ etching by the $\mathrm{NaOH}$ approach makes feasible the formation of hollow nanoshells preserving the inner SPIONs, in the form of $\mathrm{Au}$ magnetic nanorattles. 


\section{ARTICLE}
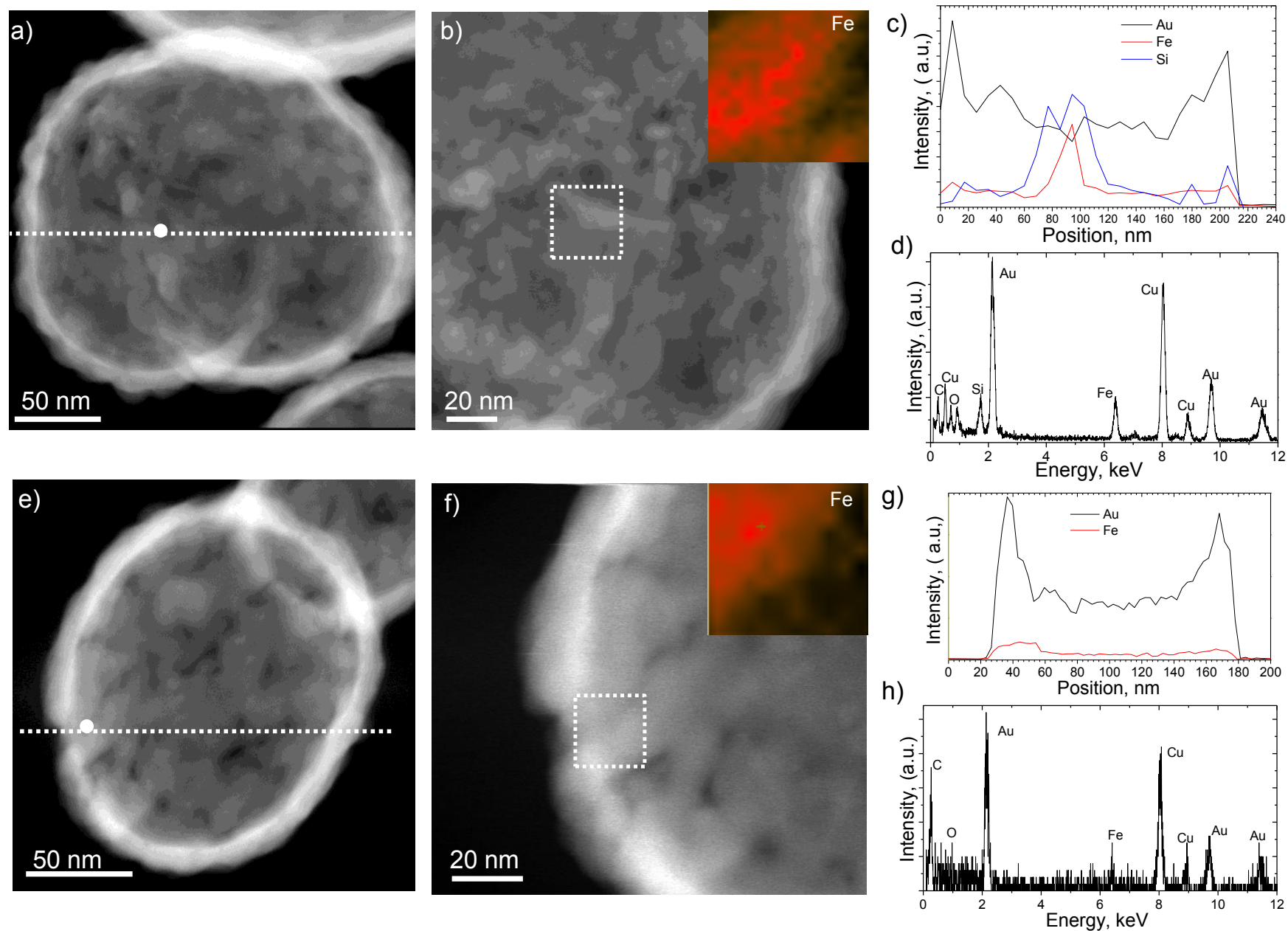

Figure 4.- Characterization of partially hollow (PH) and hollow (H) gold-magnetic nanoparticles after $\mathrm{SiO}_{2}$ etching by BHF and $\mathrm{NaOH}$ approaches. BHF etching: a) STEMHAADF image of a PH gold- magnetic nanoparticle obtained after 2 hours with a 0,001 volume of BHF/volume ratio of nanoshell dispersion. b) STEM-HAADF image of a detail area where a SPION is located under the Au shell. Inset is an Fe elemental mapping from the selected area in figure 4-b.c) Au, Si and Fe EDS profile along the selected line in figure 4-a. Highest Fe and Si signal were detected in a location shifted from the NP center. d) EDS spectra at the point selected in figure 4-a. The Si peak indicates that there is a silicon oxide layer protecting the SPION core. $\mathrm{NaOH}$ etching: e) STEM-HAADF image of a hollow gold-magnetic nanoparticle obtained after 15 minutes etching with a 1 volume of $\mathrm{NaOH}(3 \mathrm{M}) /$ volume of nanoshell dispersion. f) STEM-HAADF image of a detail area where a $\mathrm{SiO}_{2}$-SPION core-shell nanoparticle is located under the Au shell. Inset is an Fe elemental mapping from the selected area in figure 4-f. g) Au and Fe EDS profile along the selected line in figure 4-e. Fe was detected close to the gold shell. Si was not detected h) EDS spectra at the point selected in figure 4-e.

\section{Relaxivity and optical properties}

To assess the suitability of $\mathrm{H} / \mathrm{PH}$ SPION-Au nanoshells as starting material for MRI contrast agents, water based suspensions were synthesized by dispersing them in DDI water, after removal of any trace of BHF and $\mathrm{NaOH}$ etchants. To check the stability of these suspensions, prior to the relaxivity measurements, the SPION-Au nanoshells were magnetically separated in a magnetic field gradient produced by a $3 \mathrm{kOe} \mathrm{Nd-}$ $\mathrm{B}-\mathrm{Fe}$ permanent magnet. The dispersions of H/PH SPION-Au nanoshells, which were initially blue-green dark, were almost transparent after a few hours of exposure to the magnetic field gradient (see Figure 5 a-b). Once the magnet was removed from the vicinity of the dispersion vial, the nanoparticles were easily dispersed, recovering the initial state of the suspension. This fact together with the absence of agglomeration/precipitation shows the presence of the PEG coating even after the etching process, and confirms its role in preserving the stability of those suspensions. These tests show also that the hollow $(\mathrm{H})$ /partially hollow (PH) SPION-Au 


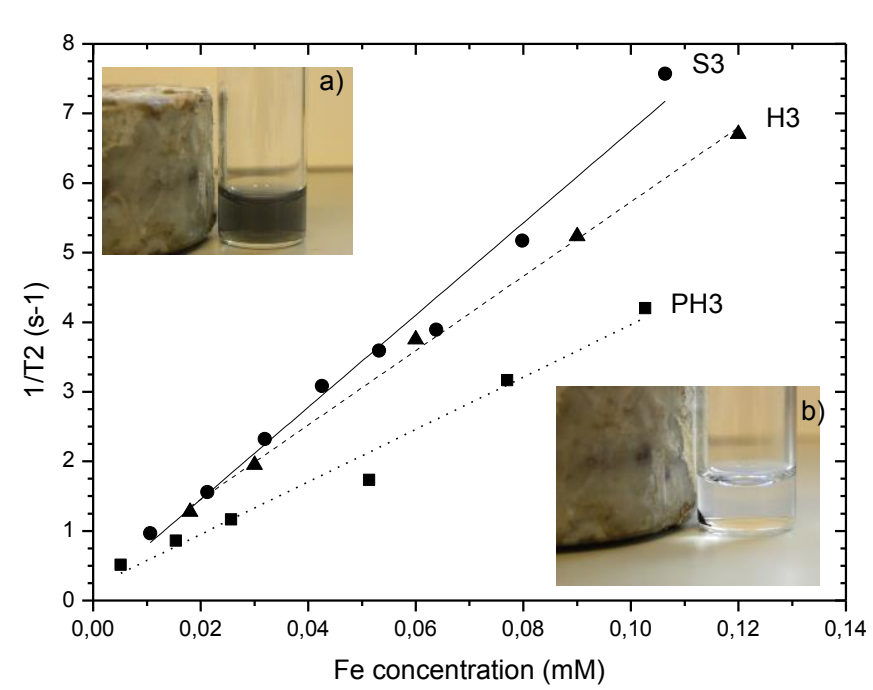

Figure 5.- $1 / T_{2}$ as a function of Fe concentration for dispersions of solid nanoshells $(\mathrm{S} 3,202 \mathrm{~nm})$ and the corresponding hollow $(\mathrm{H} 3)$ and partially hollow nanoshells (PH3), measured at 1,41T and 37으. The slopes of the lineal fit correspond to the transversal relaxivities $r_{2}$. Figure $a-b$ : hollow magnetic nanoshells (H3) before and after being placed in the magnetic field gradient produced by a Nd-B-Fe permanent magnet (at the left hand side of the image).

nanoshells can be used as cargo magnetic carriers in all those applications making use of magnetic field gradients.

The longitudinal $\left(\mathrm{r}_{1}\right)$ and transversal $\left(\mathrm{r}_{2}\right)$ relaxivity values of comercial ferrofluids (SPION), and of water based suspensions of the silica coated SPION and solid hollow and partially hollow nanoshells are presented in Table 2, The low values of $\mathrm{r}_{1}$ are in good agreement with the superparamagnetic character of the commercial fluid used for the synthesis of the $\mathrm{H}$ and $\mathrm{PH}$ nanoshells, and of the magnetic nanoparticles obtained at the subsequent synthesis steps. As previously reported by other authors, the relaxivity of core-shell nanoparticles is clearly influenced by the shell covering the magnetic core, diminishing $r_{1}$ and $r_{2}$ as the coating thickness and therefore, the distance between the magnetic core and the water protons increases. ${ }^{20-22}$ This fact explains the drastic reduction of the transversal relaxivity value taking place after coating the commercial nanoparticles by a $\mathrm{SiO}_{2}$ layer, even though in the case of silica coatings, a certain thickness of this silica shell is water permeable. ${ }^{[19,20]}$ The decrease of $r_{2}$ observed in the S3 suspension, indicates that this permeability is reduced after gold

Table 2.- Relaxivity values $\left(\mathrm{s}^{-1} \mathrm{mM}^{-1}\right)$ of the nanostructure aqueous dispersions at $37^{\circ} \mathrm{C}$ and $1.41 \mathrm{~T}$

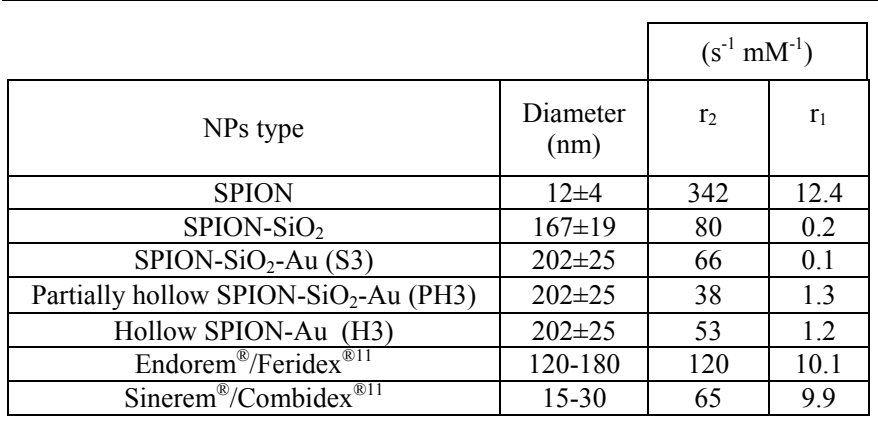

coating to obtain the SPION-SiO $2-\mathrm{Au}$ particles. In the case of the PH3 suspension, the observed decrease in $r_{2}$ can be ascribed to the before mentioned etching of the SPION core, leading to a loss of magnetic material. This loss is less pronounced in the case of the $\mathrm{H} 3$ suspension, since, as mentioned before, $\mathrm{NaOH}$ etching is milder compared to etching using BHF.

The $r_{2}$ values reported in Table 2 are of the order of those of the Sinerem ${ }^{\circledR} /$ Combidex $^{\circledR 11}$. Therefore the PH and H SPION$\mathrm{Au}$ nanoshell suspensions are potential candidates to act as $\mathrm{T}_{2}$ (or negative) contrast agents for MRI.

Figure 6 shows UV-vis-NIR optical absorbance spectra of aqueous dispersions of the engineered SPION-Au nanoshells of different $\mathrm{SiO}_{2}$ core diameter. A general trend in the etched SPIO nanoshells is that the SPR of the nanoshells blue-shifted as the $\mathrm{SiO}_{2}$ core was reduced in size by etching (e.g. from 854 $\mathrm{nm}$ to $797 \mathrm{~nm}$ in S3 and $\mathrm{H} 3$, respectively). This is consistent with the expectations, since the plasmon absorbance peak of a $\mathrm{Au}$ nanoshell shifts to shorter wavelengths as the dielectric core radius to shell thickness decreases. ${ }^{38}$ In fact, the SPR strongly depends on both the dielectric core and the dielectric embedding medium. Since in this work the dielectric embedding medium was not altered, the SPR shifting is directly correlated to the core dielectric properties. According to theoretical calculations, Peña et al. ${ }^{41}$ stated that if the Au shell thickness is kept constant, the position of the SPR peak is blueshifted as the radius of the dielectric core decreases, which is consistent with the blue shift of the SPR as the $\mathrm{SiO}_{2}$ was etched away. However, as the silica core is gradually etched the volume within the $\mathrm{Au}$ shell is filled with water and $\mathrm{SiO}_{2}$ and the optical properties will depend on the induced screening charges at the boundary between the nanoshell and the dielectric core. Then, the change in the refractive index of the core, from 1.45 (silica) to 1.33 (water), tunes the maximum of the extinction spectrum to shorter wavelengths. ${ }^{42}$ In addition, according to

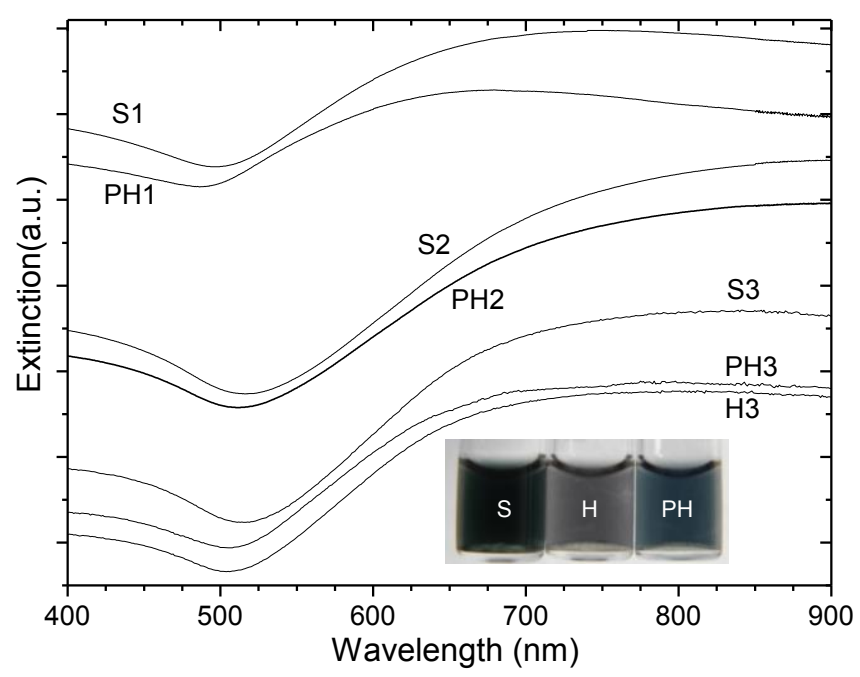

Figure 6.-UV-visible spectrum from the nanoengineered Au-SPIO nanostructures: As-made (S1), partially hollow $(\mathrm{PH})$ and hollow $(\mathrm{H})$ nanoshells. Inset.- Optical image from colloids of different Au-SPIO nanostructures. Spectra show the variation of the surface plasmon resonance band as a function of the nanoshell diameter and silica core etching grade. 
Figure 6 it seems that the SPR shifting is more notable when the non-etched nanoshell is smaller, being the shifting in PH1 higher than in $\mathrm{PH} 3$. The inset in figure 6 shows an optical image of samples $\mathrm{S} 3, \mathrm{PH} 3$ and $\mathrm{H} 3$. The etching of the core and the refractive index modification is also evident by the color change from dark greenblue (S3) to light blue grey-blue (H3).

\section{Laser heating and drug loading capability of SPION-Au nanoshells:}

Laser-induced heating tests were performed on solid, hollow and partially hollow nanoshell-based dispersions. In all the cases a significant temperature rise was observed upon irradiation. As an example, Figure 7 displays the heating efficiency of four dispersions of partially hollow PH2 and PH1 for the same dispersion concentration. PH2 shows higher heating efficiency caused by its higher absorption capacity under $808 \mathrm{~nm}$ irradiation (see Figure 6). Water was also tested as a control showing minimal temperature increases $\left(\sim 2{ }^{\circ} \mathrm{C}\right)$ under the same fluence and exposure times. Also a concentration-dependent temperature rise was observed for all the samples. The temperature rapidly increased under irradiation until reaching a plateau indicative of the balance between the energy absorbed by the plasmonic nanoparticles and the heat loss to the surroundings (air in the incubator at 37 $\left.{ }^{\circ} \mathrm{C}\right)$. Those magnetic nanoshells reached average maximum temperatures capable of inducing irreversible tissue damage ( $\Delta$ $\mathrm{T}=16.8 \pm 1.5^{\circ} \mathrm{C}$ ) in $10 \mathrm{~min}$ of exposure indicative of their potential application in photothermal therapy.

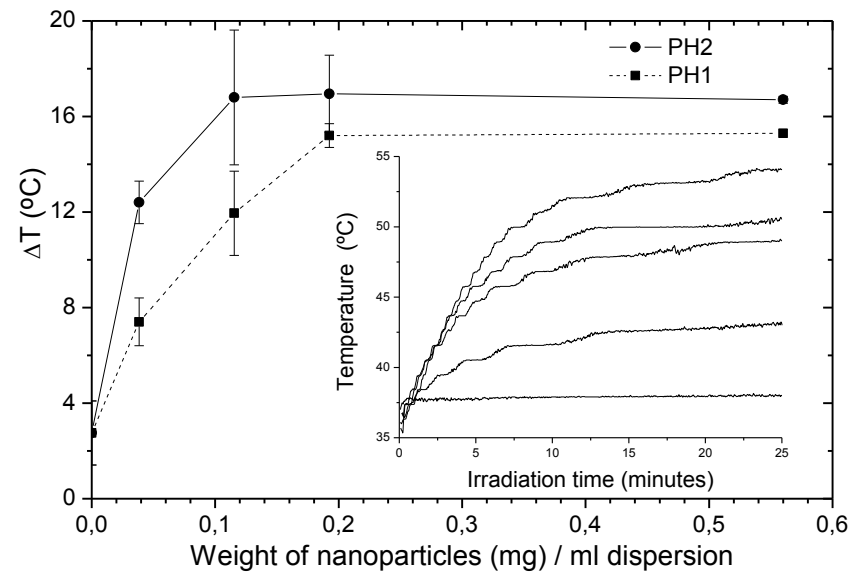

Figure 7.- a. Temperature increment for different dispersions of partially hollow nanoshells $(0,4 \mathrm{mg} / \mathrm{mL})$ irradiated with pulsed laser $(808 \mathrm{~nm})$ at $2 \mathrm{~W}$ during 25 minutes in an incubator at $37 \circ \mathrm{C}$. b. Temperature measured during 25 minutes for four dispersions $(0,04 ; 0,12 ; 0,2 ; 0,4 \mathrm{mg} / \mathrm{mL})$ of $\mathrm{PH} 1$ and of $\mathrm{PH} 2$. Water was used as control.

Drug loading and release experiments were also carried out to assess the possibility of using the nanoshells as drug delivery vectors. To this end, Rhodamine loading and release experiments were carried out for both semi-hollow and solid nanoparticles (without etching the inner silica cores). When using semi-hollow nanoparticles a Rhodamine load equivalent to $2,5 \pm 1,1$ wt. $\%(\mathrm{~N}=4)$ of the total weight of the nanoparticle was measured using UV-VIS spectrophotometry on the supernatant after adsorption, whereas no Rodamine adsorption was detected with the as-synthesized nanoparticles, which were used as control. The fact that negligible drug loading was observed for as-synthesized nanoshells seems to indicate that the adsorption of Rhodamine takes place mainly on the partially etched silica shell, a process favored by the surface roughness generated as a consequence of etching. Drug release experiments were also conducted under the presence or absence of laser light $(808 \mathrm{~nm}, 2 \mathrm{~W})$ irradiation. Given the small size of the nanoshells and the fact that only physical adsorption is involved (i.e. there is no chemical bond of the drug with the nanoshell), a rapid drug release is expected. Indeed, the results (not shown) indicate that drug release is completed within 20 min, with a fast initial period for both irradiated and nonirradiated samples; however a faster release (25\% higher in 20 min.) was observed for the irradiated samples, a fact attributed to the acceleration of desorption at higher temperatures, in agreement with previous studies ${ }^{7}$.

\section{Experimental}

\section{Materials and methods}

TEOS (tetraethyl orthosilicate, reagent grade $98 \%$ ), ammonium hydroxide (ACS reagent 28\%), formaldehyde (ASC reagent, $37 \mathrm{wt}$ \% in water), THPC (tetrakis hydroxymethyl phosphonium chloride solution, $80 \%$ in water), APTES (3Aminopropyltriethoxysilane, $>98 \%$ ), PVP powder $(\mathrm{Mw}=55000$ $\mathrm{Da}$ ), sodium hydroxide (beads 20-40 mesh), buffer fluorhydric acid (BHF), $\mathrm{HCl}$ (ACS reagent 37\%), $\mathrm{HNO}_{3}$ (ACS reagent 70 $\%$ ), $\mathrm{K}_{2} \mathrm{CO}_{3}$ (ACS reagent < 99\%), monofunctional $\mathrm{SH}-\mathrm{PEG}$ $(5000 \mathrm{Da})$ and Gold(III) chloride hydrate $\left(\mathrm{HAuCl}_{4}, 99.999 \%\right.$ trace metals basis) were purchased from Sigma Aldrich and used as received. A water based magnetite fluid (EMG 304) containing nanoparticles with a particle diameter of $11,8 \mathrm{~nm}$ was kindly donated by Ferrotech Corp (see Figure 1-a). Their magnetic characteristics were evaluated using a SQUID magnetometer, applying a d.c. magnetic field from 0 to 4 Tesla and their superparamagnetism was confirmed (results not shown). Also the temperature dependence of the magnetization was also measured by means of the so-called zerofield-cooling (ZFC) and field-cooling (FC) processes to corroborate that the nanoparticles were superparamagnetic at body temperature (results not shown).

The method proposed in this work to produce the multifunctional nanostructures comprises six sequential steps as well as the corresponding purification treatments after each step. Scheme 2 depicts the synthesis stages which can be summarized as follows: 1) Synthesis of silica magnetic coreshell NPs. 2) Surface functionalization of the silica nanoparticles. 3) Au seed grafting on the silica-magnetic coreshell NPs. 4) Au nanoshell growth. 5) PEGylation. 6) Selective silica etching.

\section{Synthesis of magnetic nanoshells}

Core/shell SPION/silica nanoparticles were synthesized following the well-known Stöber method. ${ }^{43}$ Syntheses with 
different $\mathrm{SiO}_{2}$ /SPIONs ratios were performed in order to obtain nanoparticles with different silica shells. Initially, $100 \mu \mathrm{L}$ of SPIONs $\left(\mathrm{Fe}_{3} \mathrm{O}_{4}\right.$ nanoparticles supplied by Ferrotech), $1 \mathrm{~mL}$ of DDI water and $2.5 \mathrm{mg}$ of PVP were mixed under sonication. Different amounts of the former SPION dispersion were added to $15 \mathrm{~mL}$ of absolute ethanol with $0,885 \mathrm{~mL}$ of TEOS in order to control the shell thickness. A solution with $2,665 \mathrm{~mL}$ of ammonia and $15 \mathrm{~mL}$ of absolute ethanol was separately prepared and added to the SPION dispersion under continuous mechanical stirring. The reaction was allowed to proceed at room temperature during $1,5 \mathrm{~h}$. The clear dispersion became light brown in about 1 hour. The $\mathrm{SiO}_{2}-\mathrm{SPION}$ core-shell nanoparticles formed were then surface functionalized to substitute silanol ( $\mathrm{Si}-\mathrm{OH})$ or ethoxy ( $\mathrm{Si}-\mathrm{OEt})$ groups by amino groups using silanization with APTES. The silica surface of the $\mathrm{SiO}_{2}$-SPION dispersed in absolute ethanol $(30 \mathrm{~mL})$ was treated with $30 \mu \mathrm{L}$ of APTES for 2 hours at $65^{\circ} \mathrm{C}$. After the reaction mixture had cooled down, the $\mathrm{SiO}_{2}$-SPIONs were separated from the reaction medium by centrifugation at $10.000 \mathrm{rpm}$ during 10 minutes and redispersed in ethanol. To eliminate the excess of unbound APTES, the amino-functionalized $\mathrm{SiO}_{2}$ SPIOs were ultra-centrifuged at least 3 times and redispersed in ethanol. This process was necessary since we observed that excess of APTES led to nanoparticle agglomeration, potentially caused by the interparticle hydrogen bonding between the surface silanol groups and the amine groups. ${ }^{44}$ The resulting amino-functionalized $\mathrm{SiO}_{2}$-SPION dispersion was stored for further use.

Separately, a gold-based colloid was prepared following the procedure described by Duff et al. ${ }^{45}$. In brief, $0.5 \mathrm{~mL}$ of $\mathrm{NaOH}$ (1M) and $12 \mu \mathrm{L}$ of THPC (previously dissolved in $1 \mathrm{~mL}$ of water) were dissolved in $45 \mathrm{~mL}$ of water. Afterwards, $2 \mathrm{~mL}$ of $\mathrm{AuClO}_{4}$ solution $(10 \mathrm{mg} / \mathrm{mL})$ was added to the previous solution under mechanical stirring and the dispersion turned dark brown-red. According to this protocol 2-3 nm gold nanoparticles were formed. In the next step, gold nanoparticles were covalently attached to the amino groups of the surface of the previously formed $\mathrm{SPION}-\mathrm{SiO}_{2}$ taking advantage of the strong chemical bond between nitrogen present in the amino groups and the gold orbitals via their lone pairs. ${ }^{46}$ Finally, 20 $\mathrm{mL}$ of the previous gold colloid were mixed with $2 \mathrm{~mL}$ of the functionalized silica nanoparticles and $100 \mu \mathrm{L}$ of $\mathrm{HCl}$ was added to obtain a $\mathrm{pH}$ of 2.5 which enhance the strong electrostatic interaction between the protonated amino groups of the surface of the silica and the negatively charged THPCstabilized gold nanoparticles leading to a uniform distribution of well-dispersed gold nanoparticles on the surface of the $\mathrm{SiO}_{2}$ SPIO nanoparticles. After 2 hours of reaction, the resulting mixture was ultra-centrifuged at $2000 \mathrm{rpm}$ for 30 minutes and re-dispersed in DDI water 5 times to eliminate the excess of unbound gold nanoparticles.

To grow the gold shell on the previous $\mathrm{SiO}_{2}-\mathrm{SPIO}$ nanoparticles decorated with gold seeds we followed the experimental protocol described by Pham et al. ${ }^{47}$ Firstly, $50 \mathrm{mg}$ of $\mathrm{K}_{2} \mathrm{CO}_{3}$ and $3 \mathrm{~mL}$ of gold solution ( $1 \mathrm{wt}$. \%) was dissolved in $200 \mathrm{~mL}$ of water and left for 30 minutes under continuous stirring. Then in a flask, to a $4 \mathrm{ml}$ of $\mathrm{K}_{2} \mathrm{CO}_{3}$ solution, $200 \mu \mathrm{L}$ of silica nanoparticles with the previous gold colloid and $20 \mu \mathrm{L}$ of formaldehyde were added. After 10 minutes of mechanical stirring, nanoshells with an apparent continuous gold shell were formed. The reported protocol was modified to scale-up the production of SPION-SiO $2-\mathrm{Au}$ nanoshells. The corresponding volumes of precursors were added in a reaction flask of $300 \mathrm{~mL}$ and kept under stirring during 10 minutes using an orbital shaker. In order to avoid agglomeration or even irreversible aggregation, and to increase the nanoparticle biocompatibility, $12 \mathrm{mg}$ of SH-PEG dissolved in $12 \mathrm{~mL}$ of water were added to the resulting $\mathrm{SPION}-\mathrm{SiO}_{2}-\mathrm{Au}$ and kept under stirring for 1 hour. The final PEGylated $\mathrm{SPION}-\mathrm{SiO}_{2}-\mathrm{Au}$ nanoparticles were ultracentrifuged at $3500 \mathrm{rpm}$ during 15 minutes and redispersed in water to remove un-bound SH-PEG. The resulting nanoparticles were concentrated up to $10 \mathrm{wt}$. \% and stored under refrigeration until further use.

4.3 Etching process

In order to obtain hollow gold nanospheres loaded with SPIONs the PEGylated SPION-SiO $2-\mathrm{Au}$ dispersion was subjected to an etching process using $\mathrm{NaOH}$ or $\mathrm{BHF}$ as etching agents. Both $\mathrm{NaOH}$ and $\mathrm{BHF}$ react preferentially with $\mathrm{SiO}_{2}$ although the SPIONs $\left(\mathrm{Fe}_{3} \mathrm{O}_{4}\right)$ could also be dissolved according to the following reactions:

Equation 2. $\quad \mathrm{SiO}_{2}+\mathrm{HF} \rightarrow \mathrm{SiF}_{4}(\mathrm{~g})+2 \mathrm{H}_{2} \mathrm{O}$

Equation 3. $\quad \mathrm{Fe}_{3} \mathrm{O}_{4}+\mathrm{HF} \rightarrow$ soluble iron complexes ${ }^{48}$

Equation 4. $\mathrm{SiO}_{2}+2 \mathrm{NaOH} \rightarrow \mathrm{Na}_{2} \mathrm{SiO}_{3}+\mathrm{H}_{2} \mathrm{O}$

Equation 5. $\mathrm{Fe}_{3} \mathrm{O}_{4}+4 \mathrm{NaOH} \rightarrow \mathrm{Na}_{2} \mathrm{FeO}_{2}+2 \mathrm{NaFeO}_{2}+2 \mathrm{H}_{2} \mathrm{O}$ The etching time was controlled in order to preserve the SPIONs while maximizing the silica removal. In this way, the etching process was performed using different $\mathrm{BHF}$ and $\mathrm{NaOH}$ concentrations for 2 hours and 15 minutes, respectively. Considering that both the thickness of the silica and the SPION content will influence the $\mathrm{T}_{2}$ relaxation time ${ }^{20,22,40}$, and therefore the relaxivity of water-based colloids obtained from the hollow gold nanospheres, $\mathrm{T}_{2}$ measurements (performed in Time-Domain (TD) NMR Minispec mq 60 from Bruker) and STEM-HAADF images (FEI TECNAI F30) were used to control the etching process. Then, TD NMR enables the selection of the optimal etching reagent concentration, preserving the SPIONs from etching as much as possible. Due to the high electronic contrast of Au during TEM visualization, it is not possible to observe the inner core; therefore, aqua regia was used to selectively remove the $\mathrm{Au}$ shell while preserving the silica cores in order to visualize their interiors. STEMHAADF-EDS analysis was carried out in order to identify and quantify the atomic composition of the Spion- $\mathrm{SiO}_{2}-\mathrm{Au}$ etched nanoparticles.

Hollow nanoshells with SPION cores inside were obtained by etching magnetic silica-gold nanoshells $(15 \mathrm{~mL})$ with different volumes of a $3 \mathrm{M} \mathrm{NaOH}$ solution. After 15 minutes under mechanical stirring, the resulting nanostructures were separated from the etchant solution by ultracentrifugation at $3500 \mathrm{rpm}$ for 15 minutes, and redispersed in water during 3 cycles. An alternative purification approach was developed to avoid the breakdown of the hollow nanoshells after being dispersed in the 
ultrasonic bath: given their magnetic character, they were concentrated by using a permanent magnet, and dispersed in water after magnet removal.

\section{Characterization}

Particle-size distribution and shell thickness of the resulting nanoparticles during the different synthesis steps were obtained using statistical analysis ( $>100$ particles). of TEM (T20, FEI Tecnai-200kV, at LMA-INA) images STEM imaging and EDSHAADF analysis were performed on a scanning transmission microscope (F30, FEI Tecnai-300kV, at LMA-INA). Samples for the (S)TEM observations were prepared by dispersing the nanoparticles in ethanol and evaporating suspension drops on Holey Formvar carbon-coated copper grids. UV-VIS-NIR spectra of the different nanoparticles were measured to evaluate their characteristic extinction peaks using a Jasco spectrophotometer (V670). The NIR absorbance of the plasmonic nanoshells was also evaluated in terms of heating efficiency after laser irradiation of the corresponding colloidal dispersions using a milisecond-pulsed laser $808 \mathrm{~nm}$ at $100 \mathrm{~Hz}$ (Changchun New Industries Optoelectronics, model MDLH.808/2000mW). The colloidal dispersions were placed inside an incubator with temperature control to evaluate the temperature increase at different fluences.

Drug loading was measured using UV-VIS spectrophotometry (554 $\mathrm{nm}$ ) on the supernatants by determining the concentration after adsorption of Rhodamine B isothiocyanate $(100 \mu \mathrm{g} / \mathrm{mL})$ used as a model compound. Release was evaluated at room temperature by placing the loaded dispersions on a PBS solution and following the evolution of Rhodamine $B$ isothiocianate concentration.

To quantify the efficacy of the nanoparticles to act potentially as contrast agents for magnetic resonance imaging (MRI), relaxivities $r_{1}$ and $r_{2}$, were calculated from the slope of the lineal regression fit obtained after plotting the inverse of the relaxation times versus $\mathrm{Fe}$ concentration for at least 5 dispersions of nanoparticles in water, with different nanoparticle concentrations (see Equation 1). The $T_{2}$ relaxation times were measured at room temperature, under a constant magnetic field of 1.5 Tesla, using a standard Carr-PurcelMeiboom-Gill sequence. Fe concentration was determined using atomic absorption spectroscopy (Spectra A 110 Varian Inc.)

\section{Conclusions}

The procedure developed in this work allows the synthesis of hybrid magnetite-silica-gold nanoparticles, with a good control of the morphology and relative proportions of the three phases. Depending on the conditions used, the resulting magnetoplasmonic entities can have full, hollow and partially hollow nanostructures. The synthesis is easily scalable (at least 75 times, up to a volume of $300 \mathrm{~mL}$ ) and the resulting nanoparticles maintain the magnetic and optical properties of the original core SPIONs and Au-nanoshells respectively, with relaxivity values similar to those reported for commercial
SPION-based contrast agents and good photothermal heating properties. By controlling the concentration of etching agent and the etching time it is possible to remove the interior silica while preserving the inner magnetic cores and the SPR of the $\mathrm{Au}$ shell. The hollow interior can be used to store a drug or molecules of interest in the partially etched nanoshells. The final construct has potential as a theranostic platform, combining two therapeutic possibilities (drug delivery and NIR hyperthermia) and imaging capabilities in MRI applications.

\section{Acknowledgements}

This work was supported by the Spanish Ministry of Science and Innovation (MICINN) through the MAT2011-24988 grant. The ERC (Advanced Grant HECTOR) and People Program (Marie Curie Actions) of the EU under the REA grant agreement no. 321642 are also gratefully acknowledged.

\section{Abbreviations}

$\mathrm{S}$, as-synthesized nanoshell; $\mathrm{PH}$, partially hollow nanoshell; $\mathrm{H}$, hollow nanoshell; SPR, surface plasmon resonance; NP, nanopar-ticle

\section{Notes and references}

${ }^{\dagger}$ Department of Chemical Engineering. Aragon Institute of Nanoscience (INA), University of Zaragoza, Campus Río EbroEdificio I+D, C/ Poeta Mariano Esquillor S/N, 50018-Zaragoza, Spain.

* CIBER de Bioingeniería, Biomateriales y Nanomedicina (CIBER-BBN), Campus Río Ebro-Edificio I+D, C/Poeta Mariano Esquillor S/N, 50018-Zaragoza, Spain

a Instituto de Ciencia de Materiales de Aragón (ICMA), CSICUniversidad de Zaragoza, C/ Pedro Cerbuna 12, 50009Zaragoza, Spain.

b Departamento de Física de la Materia Condensada, Universidad de Zaragoza, C/ Pedro Cerbuna 12, 50009Zaragoza, Spain.

*Corresponding authors. Emails: victorse@unizar.es, Jesus.Santamaria@unizar.es

1. E. Boisselier and D. Astruc, Chem Soc Rev, 2009, 38, 17591782 .

2. S. Jelveh and D. B. Chithrani, Cancers, 2011, 3, 1081-1110.

3. S. J. Oldenburg, R. D. Averitt, S. L. Westcott and N. J. Halas, Chem Phys Lett, 1998, 288, 243-247.

4. L. Hirsch, A. Gobin, A. Lowery, F. Tam, R. Drezek, N. Halas and J. West, Annals of Biomedical Engineering, 2006, $34,15-22$.

5. Z. Qin and J. C. Bischof, Chem Soc Rev, 2012, 41, 11911217.

6. S. R. Sershen, S. L. Westcott, N. J. Halas and J. L. West, J Biomed Mater Res, 2000, 51, 293-298.

7. C. Yague, M. Arruebo and J. Santamaria, Chem Commun, 2010, 46, 7513-7515. 
8. X. H. Huang, I. H. El-Sayed, W. Qian and M. A. El-Sayed, $J$ Am Chem Soc, 2006, 128, 2115-2120.

9. V. Cebrian, F. Martin-Saavedra, L. Gomez, M. Arruebo, J. Santamaria and N. Vilaboa, Nanomed-Nanotechnol, 2013, 9, 646-656.

10. J. W. M. Bulte and D. L. Kraitchman, Nmr Biomed, 2004, 17, 484-499.

11. C. Corot, P. Robert, J. M. Idee and M. Port, Adv Drug Deliver Rev, 2006, 58, 1471-1504.

12. Y.-X. Wang, S. Hussain and G. Krestin, European Radiology, 2001, 11, 2319-2331.

13. N. Lee and T. Hyeon, Chem Soc Rev, 2012, 41, 2575-2589.

14. J. E. Rosen, L. Chan, D.-B. Shieh and F. X. Gu, Nanomedicine: Nanotechnology, Biology and Medicine, 2012, 8, 275-290.

15. G. F. Goya, V. Grazu and M. R. Ibarra, Current Nanoscience, 2008, 4, 1-16.

16. Y. X. Wang, Quant Imaging Med Surg, 2011, 1, 35-40.

17. L. E. W. LaConte, N. Nitin, O. Zurkiya, D. Caruntu, C. J. O'Connor, X. P. Hu and G. Bao, Journal of Magnetic Resonance Imaging, 2007, 26, 1634-1641.

18. C. Vogt, M. S. Toprak, M. Muhammed, S. Laurent, J. L. Bridot and R. N. Muller, Journal of Nanoparticle Research, 2010, 12, 1137-1147.

19. D. X. Chen, N. Sun, Z. J. Huang, C. M. Cheng, H. Xu and H. C. Gu, Journal of Magnetism and Magnetic Materials, 2010, $322,548-556$.

20. S. L. C. Pinho, S. Laurent, J. Rocha, A. Roch, M.-H. Delville, S. Mornet, L. D. Carlos, L. Vander Elst, R. N. Muller and C. F. G. C. Geraldes, The Journal of Physical Chemistry C, 2011, 116, 2285-2291.

21. S. L. C. Pinho, G. A. Pereira, P. Voisin, J. Kassem, V. Bouchaud, L. Etienne, J. A. Peters, L. Carlos, S. Mornet, C. F. G. C. Geraldes, J. Rocha and M.-H. Delville, Acs Nano, 2010, 4, 5339-5349.

22. K. Tanaka, A. Narita, N. Kitamura, W. Uchiyama, M. Morita, T. Inubushi and Y. Chujo, Langmuir, 2010, 26, 11759-11762.

23. J. Kim, S. Park, J. E. Lee, S. M. Jin, J. H. Lee, I. S. Lee, I. Yang, J.-S. Kim, S. K. Kim, M.-H. Cho and T. Hyeon, Angewandte Chemie International Edition, 2006, 45, 77547758 .

24. S. I. Stoeva, F. Huo, J.-S. Lee and C. A. Mirkin, Journal of the American Chemical Society, 2005, 127, 15362-15363.

25. X. Ji, R. Shao, A. M. Elliott, R. J. Stafford, E. Esparza-Coss, J. A. Bankson, G. Liang, Z.-P. Luo, K. Park, J. T. Markert and C. Li, The Journal of Physical Chemistry C, 2007, 111, 6245-6251.

26. M. E. Khosroshahi and L. Ghazanfari, Materials Chemistry and Physics, 2012, 133, 55-62.

27. M. Chen, Y. N. Kim, H. M. Lee, C. Li and S. O. Cho, J Phys Chem C, 2008, 112, 8870-8874.

28. L. Wang, J. Bai, Y. Li and Y. Huang, Angewandte Chemie International Edition, 2008, 47, 2439-2442.

29. L. W. Melancon M.P., Li C., Mrs Bull, 2009, 34, 415-421.

30. M. P. Melancon, M. Zhou and C. Li, Accounts of Chemical Research, 2011, 44, 947-956.
31. S. Parveen, R. Misra and S. K. Sahoo, Nanomedicine: Nanotechnology, Biology and Medicine, 2012, 8, 147-166.

32. J. Gautier, E. Allard-Vannier, E. Munnier, M. Souce and I. Chourpa, J Control Release, 2013, 169, 48-61.

33. A. Kumar, X. Zhang and X.-J. Liang, Biotechnology Advances, 2013, 31, 593-606.

34. R. Singh and J. W. Lillard, Exp Mol Pathol, 2009, 86, 215223.

35. M. P. Melancon, W. Lu, Z. Yang, R. Zhang, Z. Cheng, A. M. Elliot, J. Stafford, T. Olson, J. Z. Zhang and C. Li, Mol Cancer Ther, 2008, 7, 1730-1739.

36. J. You, G. Zhang and C. Li, Acs Nano, 2010, 4, 1033-1041.

37. S. J. Oldenburg, R. D. Averitt, S. L. Westcott and N. J. Halas, Chem Phys Lett, 1998, 288, 243-247.

38. R. D. Averitt, D. Sarkar and N. J. Halas, Phys Rev Lett, 1997, $78,4217-4220$.

39. L. Gomez, V. Sebastian, S. Irusta, A. Ibarra, M. Arruebo and J. Santamaria, Lab Chip, 2014, 14, 325-332.

$40 . \quad$ S. L. C. Pinho, S. Laurent, J. Rocha, A. Roch, M. H. Delville, S. Mornet, L. D. Carlos, L. Vander Elst, R. N. Muller and C. F. G. C. Geraldes, J Phys Chem C, 2012, 116, 2285-2291.

41. O. Pena, U. Pal, L. Rodriguez-Fernandez and A. CrespoSosa, J Opt Soc Am B, 2008, 25, 1371-1379.

42. C. Graf and A. van Blaaderen, Langmuir, 2002, 18, 524-534.

43. W. Stober, A. Fink and E. Bohn, J Colloid Interf Sci, 1968, 26, 62-\&.

44. J. Lu, M. Liong, J. I. Zink and F. Tamanoi, Small, 2007, 3, 1341-1346.

45. D. G. Duff, A. Baiker and P. P. Edwards, Langmuir, 1993, 9, 2301-2309.

46. A. H. Pakiari and Z. Jamshidi, J Phys Chem A, 2007, 111, 4391-4396.

47. T. Pham, J. B. Jackson, N. J. Halas and T. R. Lee, Langmuir, 2002, 18, 4915-4920.

48. W. W. Frenier, Corrosion, 1984, 40, 176-180. 
A new set of multi-functional nanoparticles which fill the gap of existing ones has been synthesized.

This work attempts the synthesis of two types of theranostic nanoparticles with optical, magnetic and drug loading properties by engineering continuous but porous Au nanoshells on hollow/partially hollow nanostructures containing magnetic cores .

Inmaculada Urries, Cristina Muñoz, Leyre Gomez, Clara Marquina, Victor Sebastian*, Manuel Arruebo and Jesus Santamaria*

Magneto-plasmonic nanoparticles as theranostic platforms for magnetic resonance imaging, drug delivery and NIR hyperthermia applications

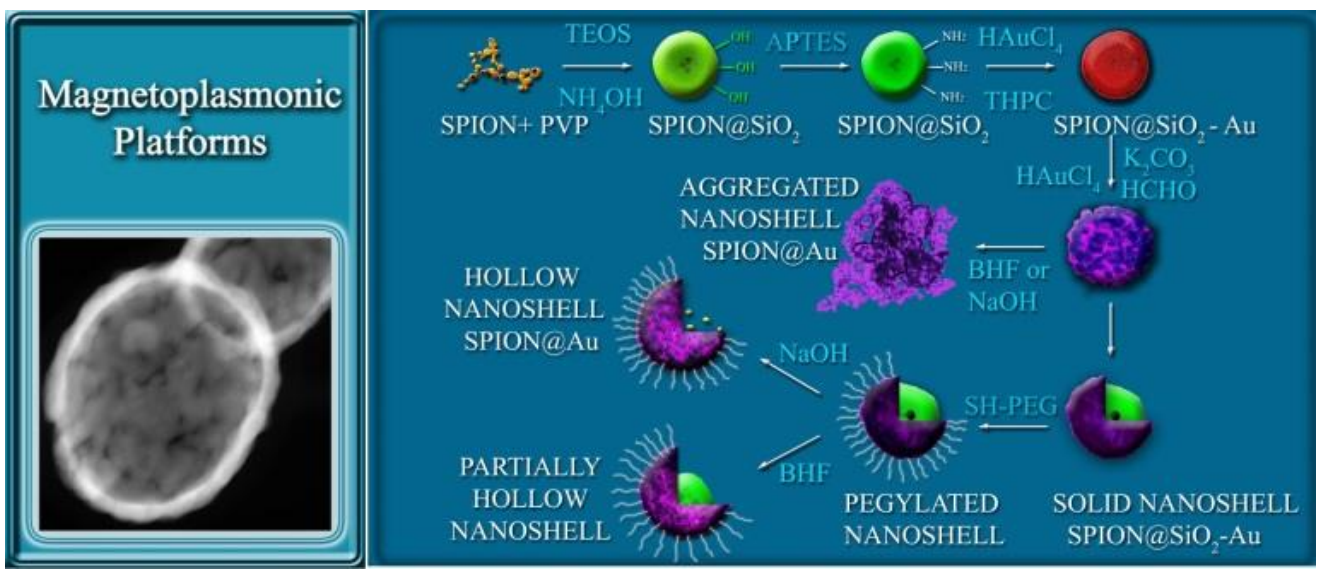

